\title{
A New Approach to Signature-Based Authentication
}

\author{
Georgi Gluhchev ${ }^{1}$, Mladen Savov ${ }^{1}$, Ognian Boumbarov ${ }^{2}$, and Diana Vasileva ${ }^{2}$ \\ ${ }^{1}$ Institute of Information Technologies, 2, Acad. G. Bonchev Str., Sofia 1113, Bulgaria \\ gluhchev@iinf.bas.bg, ihaiha99aabv.bg \\ ${ }^{2}$ Faculty of Communication Technologies, Technical University, \\ 8, Kl. Ohridski, 1000 Sofia, Bulgaria \\ olb@tu-sofia.bg, diana@engineer.bg
}

\begin{abstract}
A new signature based authentication approach is described, where signing clips are analyzed. Using an web-camera a series of frames is acquired that allows investigating the dynamics of the complex "hand-pen". For this a set of features of the hand, the pen and their mutual disposition at the time of signing is derived. Classification and verification decision-making rule based on the Mahalanobis distance has been used. A class-related feature weighting is proposed for the improvement of accuracy. A Gaussian-based model for the description of skin color is suggested. The preliminary experimental results have confirmed the reliability of the approach.
\end{abstract}

Keywords: Signature, Authentication, Biometrics, Feature weight, Classification error, Color modeling.

\section{Introduction}

The signature has been and is still used as a principal mean for person authentication. This is due to the comparative stability of the graph and of the movement dynamics stemming from the stereotype built in the years.

Until now two approaches to signature authentication have been brought to life called off-line and on-line. The development of the off-line methods started in 70-ies of the last century $[11,12]$. They are based on the evaluation of sophisticated static features describing the signature's graph $[3,6,7,9,10]$. Unfortunately, the graph can be imitated skillfully within the range of the admissible variations of the individual. This makes the identification methods using off-line analysis of the signature not quite reliable.

To speed up the authentication and increase the reliability on-line analysis has been introduced, where a pressure sensitive graphic tablet is used, thus giving the possibility for the analysis of the graph, dynamics and pressure simultaneously $[4,8]$. Since it is difficult to imitate dynamics, it is believed that this approach will be more resistant to forgeries. However, the non-standard way of signing may involve deviations in the signature parameters and may cause an increase in the error.

While the two above-mentioned approaches encompass the signature graphics, dynamics and pressure, there is one more aspect of the writing process that may contribute to the authentication problem solution. This concerns the hand position and parameters, pen orientation and its relevant position to the hand during the signing, 
i.e. the behavior of the complex "hand-pen". This aspect is described in the paper and some preliminary results are reported. To the best of our knowledge, such an investigation, where interconnected parameters of different character, reflecting the individuality of the signing subject, has not been carried out.

The paper is organized in the following way: in section 2 some preprocessing and segmentation steps are presented; section 3 describes feature extraction; section 4 introduces the authentication rule; in section 5 experimental results are presented; in section 6 some problems are discussed and the possibilities for further extension of the approach are outlined.

\section{Pre-processing and Segmentation}

The approach is based on the processing of images of a signing hand. For this the following scenario is used: the hand enters the field of a web-camera mounted above the desk, does signing and leaves the field after that. Thus, a video-clip is obtained and saved. Depending on the signature length, the series of images can contain between 100 and 200 frames.

\subsection{Image Pre-processing}

The image processing consists of several steps: detection of the signature end points, hand-pen extraction and evaluation of features related to the hand parameters and hand-pen dynamics.

\subsection{Detection of the Signature's Start and End Points}

The procedure for detection of the signature's start and end points was described in detail in [15]. The absolute difference between the frames and an empty reference frame is used for this. When the hand enters the view field of the camera this difference will rise sharply, then will oscillate about some constant value and will diminish with the hand's outdrawing, so a graph with steep slope at the beginning and at the end of the signing will be produced (Fig. 1). Calculating the values of the gradient of the graph provides an easy determination of the "plateau" and so of the beginning and the end of the signature.

The experiments carried out in [15] have shown satisfying precision in the localization of the signature. Variations of about 2 frames have been observed.

At this initial step the signature duration $\mathrm{d}$ as a number of frames can be obtained and used further as an identification feature.

\subsection{Object Detection}

The complex "hand-pen" is the object that we are interested in. Because of the almost uniform background a fixed threshold could be applied for the object's detection. To evaluate it, a few frames, say $\mathrm{K}$, are recorded in the absence of any object. For every two consecutive empty frames $(\mathrm{k}, \mathrm{k}+1)$ the maximal absolute difference $\mathrm{E}^{\mathrm{k}, \mathrm{k}+1}$ of the values of the three color components is evaluated: 


$$
E^{k, k+1}=\max \left|E^{k+1}(i, j)-E^{k}(i, j)\right|
$$

where $E^{k}(i, j)$ is the color components vector at pixel $(i, j)$ in the $k$-th image $(k=1$, $2, \ldots, \mathrm{K}-1)$ and $\mathrm{K}$ is the number of the frames in the series.

The maximal difference

$$
E=\max _{k} E^{k, k+1}
$$

is further used as a threshold for the object detection in the image sequence. After this operation, some "salt and pepper"- type noise could remain in the image (Fig.2b), but it could be easily removed using morphological or heuristic techniques.

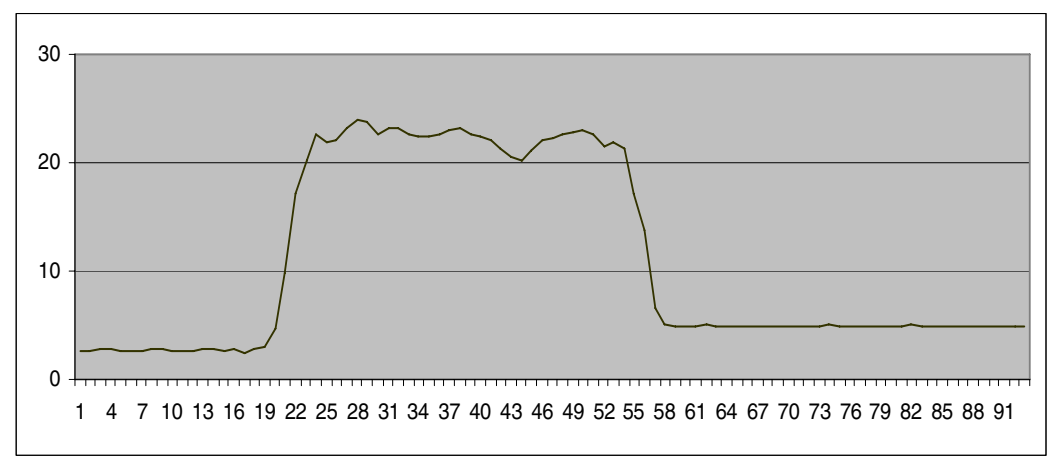

Fig. 1. Graph of the absolute differences. The horizontal axis represents the frame numbers, differences are alongside the vertical axis.

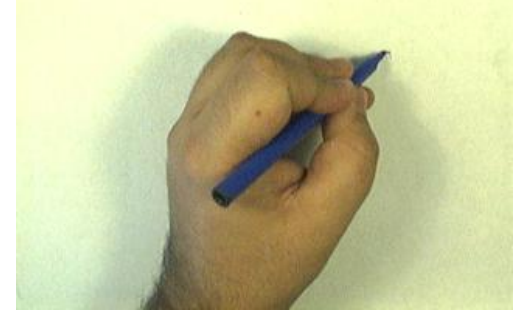

a)

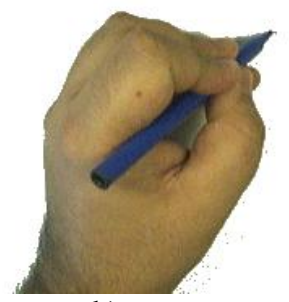

b)

Fig. 2. a) Original image; b) background subtracted

\subsection{Hand - Pen Segmentation}

For the analysis of the hand and pen movement, a separation of the two elements is required. To facilitate the task, a blue color pen is used which makes good contrast 
with the predominant skin color (red with blue and green tinges) and the background which is a sheet of white paper. The use of the "red/blue" relation in the pixels leads to a good separation of the hand from the pen (Fig. 3).

\section{3 “Hand-Pen” System Features Extraction}

The essential authentication features include hand characteristics and movement, pen position and the mutual hand-pen disposition.

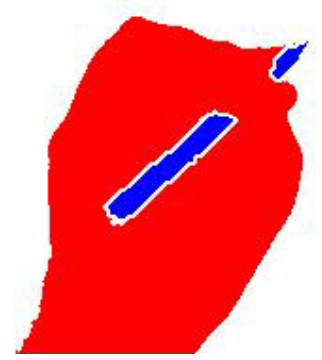

Fig. 3. Separation of the pen from the hand

\subsection{Hand Features}

Hand color and hand geometry are biometrics parameters specific for the individual and can contribute to the authentication.

Hand color. The hand color will speed up the search and increase the accuracy provided a large data-base of individuals of different races has to be searched for. To achieve this, models of the skin color of different races have to be generated. This requires a proper color space of minimal dimension to be selected, on the one hand, and the color components to be separated from the intensity, on the other hand. The $\mathrm{YCbCr}$ space is one of the possibilities studied in the literature that satisfies these requirements [2,5]. It is obtained from the RGB space using the non-linear transformation

$$
\begin{aligned}
& \mathrm{Y}=0.299 \mathrm{R}+0.587 \mathrm{G}+0.114 \mathrm{~B} \\
& \mathrm{Cb}=\mathrm{B}-\mathrm{Y} \\
& \mathrm{Cr}=\mathrm{R}-\mathrm{Y}
\end{aligned}
$$

The first component $Y$ reprisents the light intensity, i.e. it produces a gray-level image, while $\mathrm{Cb}$ and $\mathrm{Cr}$ are not influenced by the intensity change.

To describe the distribution of the skin color components, Gaussian function is usually used. Since the parameter distribution depends on the race, a Gaussian mixture model (GMM) is expected to be more adequate for an overall skin color presentation. The distribution density function of the mixture is defined as 


$$
p(x \mid \Theta)=\sum_{i=1}^{M} P(i) p(x \mid i, \Theta)
$$

where $\mathrm{P}(\mathrm{i})$ is the prior probability of the $i$-th race, and $\mathrm{p}(\mathrm{x} \mid \mathrm{i}, \Theta)$ is its density function. The parameter $\Theta$ consists of mean values and co-variances that have to be estimated from the experimental data. The Expectation Maximization proved to be a suitable technique for the evaluation of $\Theta$ in case of mixed models [1].

To demonstrate the possibility of GMM to distinguish between the races, a mixed distribution is shown in Fig. 4. For the evaluation of its parameters a data base created at the Technical University in Sofia is used, including 50 images of individuals from the white, black and yellow races. It is visible that nevertheless the three race-related areas overlap, it is possible to obtain a relatively good separation between them.

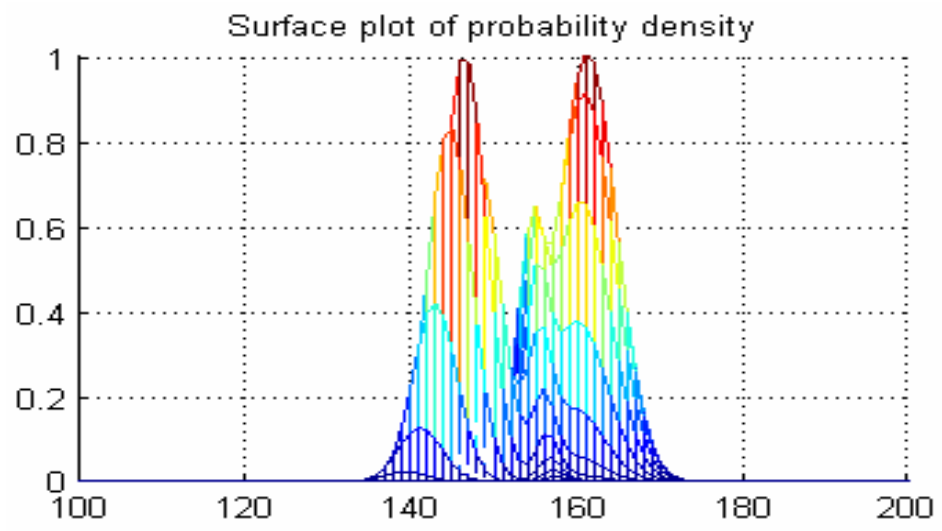

Fig. 4. Projection of a mixture of Gaussian distributions of white, black and yellow skin color. The horizontal axis represents the value of $\mathrm{C}_{\mathrm{b}}$, the probability density is alongside the vertical axis.

More sophisticated approach may use color properties of different parts of the hand, thus characterizing the individual more accurately.

Hand position and geometry. The main geometric features of the hand and its position during signing could be evaluated from its upper contour $\mathcal{K}$. scanning the image column by column and taking the coordinates $(x, y)$ of the first pixel from the hand ( $\mathrm{x}$ is the column number and $\mathrm{y}$ is the row number in the frame). The hand shape will be different for different persons because it depends on the size of the hand, on the way it holds the pen and on its movements while signing.

Hand position. The slope angle $\alpha$ of the line $1: \mathrm{y}=\mathrm{ax}+\mathrm{b}$, which approximates $\mathcal{K}$ the best way in the sense of minimal mean-square distance, could be used as a general characteristic of the hand position. For that the line parameters a and $b$ are evaluated minimizing the sum: 


$$
S=\sum_{x=0}^{M-1}[y-(a x+b)]^{2}
$$

where $(\mathrm{x}, \mathrm{y}) \in \mathcal{K}$ and $\mathrm{M}$ is the number of the contour points.

Geometric features. The geometric features are extracted using the characteristic points of the hand contour. End points and points of curvature larger than a predefined threshold are assumed as "characteristic". Different techniques could be used for curvature evaluation. To simplify the calculations, we have used the following formula

$$
c=\frac{\left|\overline{P_{-q}}\right|+\left|\overline{P_{+q}}\right|}{\left|\overline{P_{-q} P_{+q}}\right|},
$$

where $\mathrm{P}$ denotes the current point, and $\mathrm{P}_{-\mathrm{q}}$ and $\mathrm{P}_{+\mathrm{q}}$ are the points remote $\mathrm{q}$ points far from $\mathrm{P}$ in both directions. Different geometric parameters of the obtained polygon like perimeter, area or distances from its centre to specific points could be measured.

Pen position. The pen position is described by the angle $\gamma$ of its tilt towards the plane and the angle $\beta$ of its projection in the plane. Since the pen length 1 is fixed, the first angle is determined by the ratio of the projection length 1 to 1 . To determine $\beta$ and 1 ,' we have to determine the major axis of the pen. For this its centre $\left(C_{x}, C_{y}\right)$ is evaluated averaging the $\mathrm{x}$ and $\mathrm{y}$ coordinates of the pixels from the pen. After that the eigen values $\lambda_{1}$ and $\lambda_{2}$ of the characteristic equation

$$
|\Sigma-\lambda I|=0
$$

where $\Sigma$ is a co-variance matrix and $I$ is the unitary matrix is solved. $\beta$ is evaluated according to the formula

$$
\beta=\operatorname{arctg}\left(\frac{\operatorname{cov}_{11}-\lambda_{1}}{\operatorname{cov}_{12}}\right), \quad\left(\lambda_{1} \geq \lambda_{2}\right)
$$

To determine the angle $\gamma$, a straight line of angle $\beta$ is drawn through the center and the distance l' between the utmost pixels from the pen coinciding with the straight line is evaluated. $\gamma$ is obtained from the equation

$$
\gamma=\arccos \left(1^{\prime} / 1\right) \text {. }
$$

Hand-pen relative position. The mutual position of the hand and pen is described using the following parameters:

a) The difference $\delta=\alpha-\beta$

b) The distances between the pen center and hand contour. For this a straight line perpendicular to the pen's longitudinal axis and passing through the center is used. The distances $\mathrm{r}_{1}\left(\mathrm{C}, \mathrm{H}_{1}\right)$ and $\mathrm{r}_{2}\left(\mathrm{C}, \mathrm{H}_{2}\right)$ between the two cross-points $\mathrm{H}_{1}$ and $\mathrm{H}_{2}$ of that line and contour are evaluated (Fig. 5). 


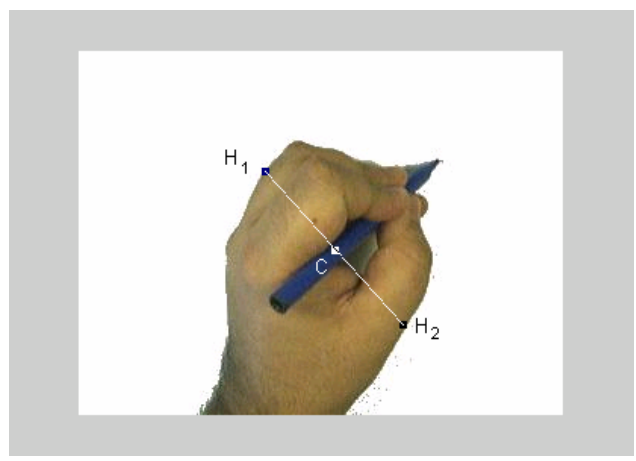

Fig. 5. Distances between the pen and hand contour

\section{Authentication}

The described in $\mathbf{3}$ set of features is measured for every individual whose signing has to be recognized. The mean value vectors $\mathbf{m}_{\mathrm{i}}$ and covariance matrices $\mathbf{S}_{\mathrm{i}}$ are evaluated and used as parameters of the squared Mahalanobis distance

$$
\mathrm{R}_{\mathrm{i}}(\mathrm{x})=\left(\mathrm{x}-\mathrm{m}_{\mathrm{i}}\right)^{\mathrm{t}} \mathrm{S}_{\mathrm{i}}^{-1}\left(\mathrm{x}-\mathrm{m}_{\mathrm{i}}\right)
$$

between the current signing $\mathbf{x}$ and the i-th individual from the data base $(\mathrm{i}=$ $1,2, \ldots, \mathrm{N})$.

There are two aspects of the authentication problem: classification and verification. While the classification problem is aimed at the assignment of a class-label to an unknown sample, the verification is aimed at the confirmation of the claimed classlabel. This difference is further used to show how the verification accuracy could be improved.

Formula (10) treats all the features equally. However, in real cases the contribution of the features may not be equal. It is more reasonable to accept that different features will have different classification value for different individuals. Therefore, it makes sense to try to evaluate feature weights for each class separately and use them as weight factors for distance evaluation. This means evaluation of class-dependent values of the features.

In this paper we show that such a class-based evaluation of feature significance is possible and may improve the decision-making. The claim is that not only the feature values characterize the individual, but also the weights of the features are specific for him.

\subsection{Feature Weighting}

The accuracy of the classification is usually evaluated in terms of correct and wrong answers. This suggests using the function $\varphi=\mathrm{Tp}-\mathrm{Fp}$ as a measure of accuracy, where $\mathrm{Tp}$ is the number of the true-positive answers and $\mathrm{Fp}$ is the number of the falsepositive ones for a particular class. The goal is to find the maximal value of $\varphi$ as a function of the feature weights $t_{i j}$ for every class $i$ and every feature $j$. Since there is 
no analytical relationship between the weights and the classification rate the only way to optimize $\varphi$ consists in varying the weights $\mathrm{t}_{\mathrm{ij}}$. A good practical scheme for this was suggested by the theory of experiment planning [14]. The gradient for the surface $\varphi$ is evaluated and the search goes in its direction until a maximal value of $\varphi$ is obtained.

\section{Experimental Results}

To test the described approach, 14 volunteers have taken parts in the experiments. 10 signatures have been acquired from each of them in different days and at different time of the day. In this study the following 8 features have been measured and used for the evaluation of $\mathbf{m}_{\mathrm{i}}$ and $\mathbf{S}_{\mathrm{i}}:$ 1) signature length $\mathrm{d}$ as a number of frames, 2) hand slope $\alpha, 3)$ pen projection angle $\beta, 4$ ) pen slope $\gamma, 5$ ) difference $\delta=\alpha-\beta, 6$ ) ratio $\mathrm{r}_{1} / \mathrm{r}_{2}$ of the distances between the pen centre and hand contour, 7) perimeter $\mathrm{p}$ of the polygon defined by the characteristic points of the upper hand contour, and 8) area of the polygon. The feature "skin colour" was not used because all the volunteers were from the same race.

\subsection{Verification}

Using the Matlab random number generator 1000 signatures have been simulated for every individual. Assuming independent features the Mahalanobis distance was evaluated according to the formula

$$
\mathrm{R}_{\mathrm{ik}}=\sum_{\mathrm{j}=1}^{8} \mathrm{t}_{\mathrm{ij}}\left(\mathrm{f}_{\mathrm{kj}}-\mathrm{m}_{\mathrm{i}}\right)^{2} / \sigma_{\mathrm{i}}{ }^{2}
$$

Moving in the gradient direction the best values of $\mathrm{t}_{\mathrm{ij}}$ in terms of maximal classification rate have been determined.

The verification results are shown in Table 1. Some individuals (Nos 3, 8, 9, 11 and 14) are not included in the table because an absolute score of $100 \%$ was achieved for them. The first three rows include the values of Tp, Fp and $\varphi$ in case of weights equal to 1 . For the next rows the above described scheme was applied. It is seen that there is an improvement of the classifier performance for all the individuals, except No 10, where no effect is observed. The most significant improvement is obtained at Nos 2 and 7. Thus, an average accuracy $\varphi=99.9 \%$ was achieved after the modification of the weights, compared to the initial accuracy of $99.6 \%$. The highest rate of false-positive $\mathrm{Fp}=0.4 \%$ was observed at the individual No 1 . The best reduction in Fp was obtained for No 2, where the accuracy increased from $99.2 \%$ to $100 \%$.

It was interesting to see how the evaluated weights would affect the results if applied to a classification problem. Using again 1000 simulations per class and the evaluated weight factors for the classes no significant changes have been observed. Almost the same average accuracy was achieved resulting in $0.02 \%$ increase in $\mathrm{Tp}$ and $0.04 \%$ decrease in Fp. Therefore, for the classification problem there is may be no need to evaluate class-dependent feature weight factors. 
Table 1. Experimental results

\begin{tabular}{|c|c|c|c|c|c|c|c|c|c|c|}
\hline weights & score & 1 & 2 & 4 & 5 & 6 & 7 & 10 & 12 & 13 \\
\hline \multirow{3}{*}{$\begin{array}{l}\text { initial } \\
\text { values }\end{array}$} & $T p$ & 999 & 999 & 997 & 996 & 994 & 990 & 998 & 1000 & 999 \\
\hline & $F p$ & 6 & 17 & 0 & 1 & 2 & 0 & 1 & 1 & 0 \\
\hline & $\varphi_{i}$ & 993 & 982 & 997 & 995 & 992 & 990 & 997 & 999 & 999 \\
\hline \multirow{3}{*}{$\begin{array}{l}\text { new } \\
\text { values }\end{array}$} & $T p$ & 999 & 1000 & 998 & 999 & 998 & 999 & 998 & 1000 & 1000 \\
\hline & $F p$ & 4 & 1 & 0 & 0 & 0 & 0 & 1 & 0 & 0 \\
\hline & $\varphi_{f}$ & 995 & 999 & 998 & 999 & 998 & 999 & 997 & 1000 & 1000 \\
\hline & $\varphi_{f}-\varphi_{i}$ & 2 & 17 & 1 & 4 & 6 & 9 & 0 & 1 & 1 \\
\hline
\end{tabular}

\section{Conclusion}

A new signature based authentication approach that takes into account the dynamics of the complex "hand-pen" is suggested. It allows evaluating specific parameters of the hand and pen and their mutual position. While the acquired source information allows for the extraction of many features of different nature, only a small number of global features and a simple classification rule have been used in the investigation. Nevertheless, the obtained tentative results aimed at the illustration of the approach, have shown quite satisfactory results. The assumption that the use of class-specific feature weights will improve the overall accuracy in case of verification has proven to be correct. The intuitive explanation of this is that if a sample does not belong to a particular class its features will not be properly weighted when a distance to that class will be measured and, as a result, larger distance value will be obtained.

The future work will be aimed at the thorough analysis of the parameter dynamics. Also, the acquisition of more experimental data will be of primary importance.

A possible extension of the approach may include different alternatives like: using a tablet instead of paper or capturing signature after signing and processing it in offline mode. Thus, more accurate dynamic and/or static information will be obtained.

Acknowledgments. The investigation was supported by BioSecure Network of Excellence of the 6th European Framework Program, contract No 507634, and the Ministry of Education and Sciences in Bulgaria, contract No 1302/2003.

\section{References}

1. Bouguila, N., Ziou, D.: Dirichlet-based probability model applied to human skin detection. In: Proc. Int. Conf. ASSP'04, vol. V, pp. 521-524 (2004)

2. Boumbarov, O., Vassileva, D., Muratovski, K.: Face extraction using 2D color histograms. In: XL Int. Scientific Conf. on Information, Communication and Energy Systems and Technologies (ICEST'2005), Nis, Serbia and Montenegro, vol. 1, pp. 334-337 (2005)

3. Fang, B., Leung, C.H., Tang, Y.Y., Kwok, P.C.K., Tse, K.W., Wong, Y.K.: Off-line signature verification with generated training samples. IEE Proc. Vis. Image Signal Process 149(2), 85-90 (2002)

4. Fink, G., Wienecke, M., Sagerer, G.: Video-Based On-Line Handwriting Recognition. In: Proc. Int. Conf. on Document Analysis and Recognition, pp. 226-230. IEEE, Los Alamitos (2001) 
5. Fu, Z., Yang, J., Hu, W., Tan, T.: Mixture clustering using multidimensional histograms for skin detection. In: Proc. of the 17th Int. Conf. on Pattern Recognition (ICPR'04), pp. 549-552 (2004)

6. Hairong, L., Wenyuan, W., Chong, W., Quing, Z.: Off-line Chinese signature verification based on support vector machines. Pattern Recognition Letters 26, 2390-2399 (2005)

7. Ismail, M.A., Gad, S.: Off-line arabic signature recognition and verification. Pattern Recognition 33, 1727-1740 (2000)

8. Jain, A.K., Griess, F.D., Connell, S.D.: On-Line Signature Verification. Pattern Recognition 35(12), 2963-2972 (2002)

9. Ka, H., Yan, H.: Off-line signature verification based on geometric feature extraction and neural network classification. Pattern Recognition 30(1), 9-17 (1996)

10. Kai, H., Yan, H.: Off-line signature verification using structural feature correspondence. Pattern Recognition 35, 2467-2477 (2002)

11. Kuckuk, W., Rieger, B., Steinke, K.: Automatic Writer Recognition. In: Proc. of Carnahan Conf. on Crime Countermeasures, Kentuky, pp. 57-64 (1979)

12. Lantzman, R.: Cybernetics and forensic handwriting investigation, Nauka, Moscow (1968)

13. Munich, M.E., Perona, P.: Camera-Based ID Verification by Signature Tracking. In: Burkhardt, H.-J., Neumann, B. (eds.) ECCV 1998. LNCS, vol. 1406, pp. 782-796. Springer, Heidelberg (1998)

14. Nalimov, V.V., Chernova, N.A.: Statistical Methods in Experiment Planning, Nauka, Moskow (1965)

15. Savov, M., Gluhchev, G.: Automated Signature Detection from Hand Movement. In: Proc. of CompSysTech'04, Rousse, Bulgaria, pp. III.A.3-1-III.A.3-6 (2004) 